Horizons philosophiques

\title{
Glenn Gould : « archilecteur » des Variations Goldberg
}

\section{Ghyslaine Guertin}

Volume 1, numéro 2, printemps 1991

Sémiotiques 2 : théories et champs d'application

URI : https://id.erudit.org/iderudit/800873ar

DOI : https://doi.org/10.7202/800873ar

Aller au sommaire du numéro

Éditeur(s)

Collège Édouard-Montpetit

ISSN

1181-9227 (imprimé)

1920-2954 (numérique)

Découvrir la revue

Citer cet article

Guertin, G. (1991). Glenn Gould : « archilecteur » des Variations Goldberg.

Horizons philosophiques, 1(2), 81-102. https://doi.org/10.7202/800873ar d'utilisation que vous pouvez consulter en ligne.

https://apropos.erudit.org/fr/usagers/politique-dutilisation/ 


\section{Glenn Gould : «archilecteur» des Variations Goldberg}

II s'agit en bref d'une musique qui n'observe ni commencement ni fin [...], une musique qui, à l'instar des amants de Baudelaire, repose, légère et sans entrave, sur les ailes du vent.

Glenn Gould'

Quelle étrange légèreté ! Un édifice sonore de trente variations où règnent la rigueur, l'équilibre, la symétrie, la cohérence harmonique. En tête et en queue des variations, l'Aria avec son thème tout à fait anodin qui proviendrait du petit livre Notenbüchlein d'Anna Magdalena Bach. Conformément à la règle baroque, contrastes et diversités de genres, de procédés d'écriture et de modes d'expression accompagnent ce complexe architectural qui repose sur une structure ternaire. L'introduction se compose d'une première triade (Aria et variations 1,2). La troisième variation inaugure un cycle de canons nos $3,6,9,27$, qui utilisent tous les intervalles depuis l'unisson jusqu'à la neuvième : les numéros $3 n+1$ s'apparentent à la danse, et

1. Glenn Gould, Contrepoint à la ligne, Écrits 11 réunis, traduits et présentés par Bruno Monsaingeon, Paris, Fayard, 1985, p. 11. 
les morceaux $3 n+2$ sont des variations de virtuosité. La conclusion est fondée, à partir du modèle de l'introduction, sur une autre triade (dernière variation, Quodlibet, et reprise de l'Aria initiale). Michel Butor, qui a si bien compris le principe de la variation, les a désignées "couronne de fleurons", "cristaux équidistants" ${ }^{2}$. C'est dans cette réserve féconde que le claveciniste Goldberg, élève de J. S. Bach, aurait puisé ses ressources afin de calmer les nuits d'insomnie du comte Herman de Kayserling, choisissant ça et là, au hasard, selon son inspiration et pour son plaisir, quelques-unes des variations.

Or, cette musique qui n'observerait ni commencement ni fin, ainsi conçue probablement pour le plaisir et la délectation des connaisseurs, renvoie métaphoriquement, dans le cas de Glenn Gould, à un commencement et à une fin. Comme deux pylônes extrêmes, elle encadre sa carrière. Une première version, produite chez Columbia ${ }^{3}$ en 1955, marque la naissance de sa carrière d'enregistrement qui s'achève avec une dernière version en 19814, quelque temps avant sa mort. Entre la naissance et la mort, l'œuvre accompagne l'interprète à Moscou 5 , Salzbourg 6 , et com-

2. Michel Butor, Dialogue avec 33 variations de Ludwig van Beethoven sur une valse de Diabelli, Paris, Gallimard, 1971, p. 57.

3. II s'agit de l'enregistrement ML $\mathbf{5 0 6 0 .}$

4. Cette version enregistrée chez CBS porte le no IM 37779. Gould n'avait pas l'habitude de graver une seconde interprétation de la même couvre. II avait cependant produit un second enregistrement en 1970, de la Sonate de Mozart no 10, en Do M. K V 330 (M 31073, The Mozart Piano Sonatas, Vol. 3), qu'il avait déjà réalisé en 1958 (ML 5274). De même en février 1981, quelques mois avant la reprise des Goldberg, avait-il gravé un second enregistrement de la Sonate de Haydn no 59 en mi bémol majeur (I2M 36947, IM 37559) qu'il avait produit aussi en 1958 (ML 5274). On se rappellera que Gould est mort le 4 octobre 1982.

5. II s'agit de l'enregistrement LDX 78799-1986, Conservatoire de Moscou, mai 1957.

6. II s'agit de l'enregistrement CMG-1986, Salzbourg, août 1959. 
bien de fois encore sur cette scène où il salua un jour définitivement? ${ }^{7}$.

L'interprétation ne saurait toutefois être répétitive. La même œuvre parcourue, comme le fait Gould, dans un sens, dans l'autre sens, dans tous les sens, n'est jamais non plus tout à fait la même. À chaque reprise surgit l'inédit, le non-dit, le nouveau. La tâche de l'exécutant consiste ainsi à traduire, à commenter, à communiquer un texte qui, conçu pour la réplique, possède comme un langage, une organisation, une syntaxe et une signification propres. L'activité de cet exécutant suppose alors un déplacement de sens d'une langue vers une autre. En jouant la partition, en se déclarant et en se mettant en jeu pour faire sens, il est par conséquent tenu de donner l'équivalent du sens tel qu'il s'inscrit dans les traces produites par le compositeur. Comment expliquer ce sens qui se cultive dans la différence? Comment fonctionne de manière plus générale l'interprétation musicale? La première partie de l'analyse qui suit tente de le démontrer en se référant au cadre théorique de la sémiologie de la musique développée par Jean Molino et Jean-Jacques Nattiez ${ }^{8}$. Cette approche fournira les éléments nécessaires afin d'expliquer, dans la seconde partie, le processus qui se cache derrière l'interprétation de Glenn Gould et qui contribue à le désigner "archilecteur" des Goldberg.

Selon Jean Molino', l'œuvre musicale, à l'instar des

7. On sait que Gould quitte définitivement la salle de concert pour se consacrer uniquement à l'enregistrement dès 1964, après neuf années de carrière internationale.

8. J. J. Nattiez, Musicologie générale et sémiologie, Paris, Christian Bourgois éditeur, 1987.

9. Jean Molino, «Fait musical et sémiologie», dans Musique en jeu, $n^{\circ} 17$, janvier 1975, p. 37-63. 
autres œuvres et actions humaines, revêt un caractère symbolique. Elle résulte d'un processus créateur (la poïétique), comporte une trace matérielle qui en manifeste l'existence (la partition), et donne lieu à diverses stratégies perceptives (l'esthétique) :

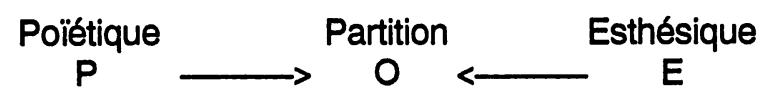

De façon générale, la poïétique ${ }^{10}(P)$ concerne la stratégie avec laquelle un créateur parvient à réaliser une œuvre quelle qu'elle soit. Cette stratégie comporte une série de facteurs de nature différente soit matérielle, psychologique, sociologique, esthétique ou philosophique. D'autre part, la trace physique ( 0 ) qui résulte de ce processus décrit l'œuvre dans sa réalité matérielle et formelle. Molino désigne cette trace de niveau matériel ou encore de niveau neutre. Enfin, l'esthétique (E) décrit les processus de réception de l'œuvre qui conduisent à la re-construction de sa signification. C'est du côté de ce pôle que se situent les différentes activités de lecture, d'analyse et d'interprétation de l'œuvre.

L'exécutant-interprète, premier lecteur de l'œuvre, se trouve donc, comme l'illustre le schéma ci-dessous, en situation de réception $\left(E_{1}\right)$ face à la partition mais également de production $\left(\mathrm{P}_{2}\right)$ par rapport à l'objet $\left(\mathrm{O}_{2}\right)$ (interprétationenregistrement) qu'il réalise. Du même côté de la réception, prend place l'auditeur $\left(E_{2}\right)$ qui est aussi en situation de production $\left(P_{3}\right)$ par rapport à sa propre représentation $\left(\mathrm{O}_{3}\right)$ de l'œuvre, et ainsi de suite...

Compositeur Interprète Auditeur $P_{1} \rightarrow O_{1}<-E_{1} \rightarrow P_{2} \rightarrow O_{2}<-E_{2} \rightarrow P_{3} \rightarrow O_{3}<-E_{3}$ etc.

10. Pour une définition complète des composantes de la tripartition, voir Nattiez, Jean-Jacques, op. cit., p. 34-40. 
Cette chaîne de communication présente donc trois types de producteurs : le compositeur, l'interprète, l'auditeur. Chacun, à des niveaux différents et avec sa propre poiétique $\left(P_{1}, P_{2}, P_{3}\right)$, collabore à la signification inépuisable d'une œuvre en la construisant et en se la représentant différemment $\left(\mathrm{O}_{1}, 0_{2}, O_{3}\right)$.

Le compositeur qui occupe le premier anneau de cette chaîne continue, détient d'emblée une place privilégiée. Son texte est en effet à l'origine des deux autres représentations, celle de l'interprète et celle de l'auditeur. L'interprète, quant à lui, fait advenir à l'existence le texte du compositeur. II se situe au centre de la chaîne et en assure en fait la continuité en transmettant au troisième anneau, occupé par l'auditeur, les significations qui proviendraient du premier anneau. Cette situation d'intermédiaire et le rôle de subordination qui revient à l'interprète par rapport à ce qu'il interprète, rappellent les composantes de cet autre schéma illustrant l'hypothèse de l'inspiration inscrite dans le dialogue entre Socrate et lon ${ }^{11}$, appliquée à la poésie. En effet, les trois anneaux sont occupés par le poète Homère, son interprète, c'est-à-dire le rhapsode lon, et l'auditeur. Un autre élément, La Muse, illustre l'origine du lien créateur qui les réunit tous les trois. Toutefois La Muse ne fait pas partie de cette chaîne. Elle est comparée à une "pierre magnétique" qui "par elle-même fait qu'en certains hommes est la Divinité12". Ainsi, comme l'a montré Michel Charles, en commentant le schéma qui suit, "la Muse inspire-t-elle le poète qui inspire le rhapsode, qui inspire l'auditeur ${ }^{13}$ »:

(La Muse) $\rightarrow$ le poète $\rightarrow$ le rhapsode $\rightarrow$ l'auditeur

11. Platon, lon, dans CEuvres complètes, Paris, Gallimard, 1950, p. 57-72.

12. Platon, ibid., 533 e).

13. Michel Charles, Rhétorique de la lecture, Paris, Éditions du Seuil, 1977, p. 74 . 
Le poète occupe dans cette chaîne, la place privilégiée du premier anneau. II est intéressant de noter que Socrate le désigne également interprète mais d'une pensée différente de celle que le rhapsode aura à interpréter. Le poète est en effet interprète de la pensée qui vient des dieux. Le rhapsode, quant à lui, est «interprète d'interprètes ${ }^{14}$ ". En d'autres termes, il est l'interprète "de ce que disent les poètes ${ }^{15}$ ". Et le rhapsode ne saura bien parler du poète que s'il est habité par cette puissance et cette grâce divine qu'est l'inspiration. Enfin, l'auditeur pour Socrate serait celui qui recevrait "les uns des autres le pouvoir à eux communiqué par la pierre d'Héraclée ${ }^{16}$ ".

Si l'on poursuit la comparaison avec les éléments de l'œuvre musicale, l'interprète serait donc lui aussi un "interprète d'interprètes". La partition n'étant toujours qu'un schéma et un produit de l'interprétation du compositeur par rapport à l'œuvre telle qu'il l'a pensée et imaginée. Sa reproduction par l'exécution ne saurait alors être comprise en tant que simple répétition, imitation ou copie de l'original. La partition requiert une pluralité d'interprétations parce qu'elle contient une pluralité de significations. Néanmoins, l'exécutant demeure toujours lié et relié à ce texte dont il doit préserver l'identité. Il est tenu en effet de communiquer un sens qui soit "l'équivalent" de celui qu'il contient. Mais toute interprétation si fidèle soit-elle, ne livre jamais la réalité intime et le secret ultime de l'œuvre. II serait illusoire de croire, à la manière d'une pensée idéaliste, que l'exécutant a non seulement le devoir mais le pouvoir d'exprimer les intentions réelles et l'esprit du compositeur : "Aucune accumulation de recherches historiques ne peut faire

14. Platon, ibid., 535 a).

15. Ibid.

16. Ibid., 535 e). 
qu'une exécution semble rendue dans un style vrai17". Cette affirmation de la part d'interprètes qui ont examiné tour à tour les questions de l'instrument, de l'articulation, des tempi, du rythme, afin de se rapprocher le plus possible dans son exécution de la pensée de Mozart et de ses intentions, est tout à fait éloquente et combien convaincante lorsqu'ils écrivent encore :

À supposer que nous puissions reproduire les mêmes conditions acoustiques, en faisant par exemple jouer des instruments anciens dans une salle de concert baroque, nous n'obtiendrons pas pour autant une audition historiquement fidèle. D'autres éléments encore devraient revenir à ce qu'ils étaient du temps de Mozart : les canons esthétiques, l'ambiance sociale ${ }^{18}$.

En d'autres termes, linterprète d'une œuvre ancienne est toujours appelé à faire un compromis, dans ce va-etvient entre son "érudition historique et l'univers réel de ses perceptions ${ }^{19}{ }^{\prime}$. C'est aussi cette implication du sujet que J. J. Nattiez retient dans son essai sur l'interprétation de Wagner lorsqu'il affirme :

Énoncer "ce que Wagner voulait dire», ce n'est jamais lire directement ses intentions dans le texte littéraire et musical qu'il a laissé, mais construire des hypothèses sur ces intentions à partir des traces que nous lisons ${ }^{20}$.

Or, ces hypothèses, que construit l'exécutant dans son parcours des traces de la pensée du compositeur,

17. Paul \& Eva, Badura-Skoda, L'art de jouer Mozart au piano, Paris, Buchet/ Chastel, 1980, p. 16.

18. Ibid., p. 17.

19. Ibid., p. 19.

20. J. J. Nattiez, Tétralogies Wagner, Boulez, Chéreau, Paris, Christian Bourgois éditeur, 1983, p. 20-21. 
découlent notamment des connaissances qu'il a de la poïétique du compositeur, de sa production, du contexte sociohistorique de la production de l'œuvre. À cette dimension se greffe également la connaissance qu'il a de l'œuvre ellemême et des autres interprétations de la même œuvre. L'activité de l'interprète, qui consiste à montrer certains traits de l'œuvre, à en cacher d'autres, à en révéler de nouveaux, n'est donc jamais neutre. Elle comporte des choix, des partis-pris qui découlent encore d'une autre série de facteurs qui appartiennent aussi à sa propre poïétique et qui sont reliés à sa formation, à son contexte de production, à sa situation historique et sociale.

On voit dès lors se dessiner le type de communication mise en branle par cette circulation continue de producteurs (émetteurs) et de récepteurs, en quête du sens de l'œuvre et de son caractère toujours inaccessible et inépuisable. Cette communication nous éloigne du contexte platonicien qui suppose la circulation linéaire d'une seule et même inspiration, du premier au dernier anneau. Et dans un sens plus réaliste, elle ne saurait non plus être conforme au schéma tout aussi linéaire qui suppose que le message est reçu comme il est émis21:

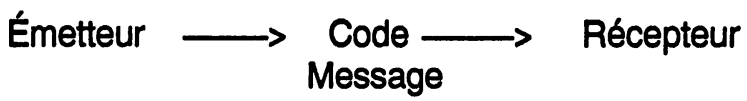

Le concept de poïétique dans la tripartition de Molino, comme l'illustre le schéma à la page 84 , conduit plutôt à démontrer l'hétérogénéité des intentions, entre compositeur, interprète et auditeur. Tous les trois projettent sur l'œuvre des configurations de sens différents, en la construisant et la re-construisant selon leurs propres points de vue. Dans ce cas, il vaut mieux admettre le malentendu et le contresens en tant que forme possible de communication requise par l'objet symbolique :

21. J. J. Nattiez, Musicologie générale et sémiologie, op. cit., p. 38. 
On ne peut écouter et même interpréter de la musique sans faire d'abord un contresens sur ce que le compositeur voulait faire. II y a là un contresens nécessaire et fondamental qui est la condition même du fonctionnement de l'objet musical?2.

II revient alors à la sémiologie d'expliquer ces contresens et ces malentendus tout en démontrant qu'il ne saurait y avoir signification d'un objet quelconque sans la médiation d'un sujet qui situe cet objet en relation avec les dimensions de son expérience. II lui appartient donc de décrire les liens, les relations que l'exécutant tisse avec la poïétique du compositeur, l'œuvre elle-même, et sa réception soit par d'autres interprètes, soit par le public. II lui incombe la tâche de démontrer que cette activité est inséparable des éléments qui composent sa propre poïétique : dimension qui contribue à définir l'exécutant comme un véritable créateur.

Si le débat actuel sur la scène musicale concerne, comme l'a montré Guy Scarpetta ${ }^{23}$, davantage l'interprétation d'œuvres anciennes plutôt que la création d'œuvres nouvelles, il n'y a pas lieu en effet de conclure sur les signes d'une crise ou les symptômes d'une décadence. Les questions qui alimentent ce débat (doit-on aborder le passé depuis le présent ou s'en remettre à la relecture historique des partitions ${ }^{24}$ ?) renvoient à la dimension recréatrice toujours présente dans l'interprétation. II s'agit là d'un objet esthétique offert à un auditeur susceptible de prendre plaisir à écouter aussi bien les Goldberg par Glenn Gould que par Wanda Landowska. Plaisir dans la recherche de la "vraie" interprétation, de l'interprétation "vraie" toujours cependant inaccessible. C'est donc cette dimension créatrice de l'interprétation qu'il s'agit d'illustrer dans

22. Jean Molino, “Apologie du contresens", Musique en Jeu, n 31, 1984.

23. Guy Scarpetta, L'impureté, Paris, Bernard Grasset, 1985, p. 47-48.

24. Ibid., p. 48. 
la seconde partie qui suit. II ne s'agit pas d'analyser, d'un point de vue sémiologique, l'une des interprétations des Goldberg par Gould, ou de comparer 25 les interprétations qu'il a gravées de cette œuvre, ni encore de démontrer l'aspect de nouveauté qu'il lui aurait apporté en cherchant davantage, comme l'affirme Scarpetta, à la "surcoder" plutôt qu'à la "décoder»26. L'objectif consiste plutôt à examiner ce qui se cache derrière cette activité créatrice de Gould lorsqu'il s'agit des Goldberg. Cet exemple constitue un objet privilégié d'observation puisqu'il permet d'avoir accès à la description d'une stratégie créatrice qui consiste en un processus de lecture précédant la réalisation sonore du texte offerte à l'auditeur. II peut sembler périlleux d'utiliser un concept emprunté à la théorie littéraire afin de l'appliquer dans le domaine de la musique. On met effectivement en présence, comme l'a démontré encore la sémiologie de la musique, deux systèmes de signes complètement différents. Par ailleurs, l'utilisation du concept de lecture en rapport avec le texte musical ou la partition n'est pas de nature métaphorique. Le terme apparaît notamment dans l'une des disciplines de base du musicien, la "lecture à vue" qui consiste à déchiffrer les signes de la partition. Elle vise plus précisément à développer la relation entre les dimensions visuelle et tactile, en cherchant à localiser la hauteur des sons sur l'instrument, à identifier les lignes mélodiques et harmoniques, à préciser la dimension rythmique. Espace et temps constituent les fondements de cet apprentissage. D'autre part, l'emprunt de ce concept aux différentes théories littéraires renvoie à une pluralité de définitions mettant alors en présence des types de lecteurs

25. Nous avons déjà effectué cette analyse avec le Groupe de Recherches Musicales de I'INA à Paris. Après avoir fait l'objet de cinq émissions à la radio de France-Culture, notre analyse a été publiée sous le titre : "À propos de "Glenn Gould et les Variations Goldberg" : une approche sémiologique de l'interprétation", in Analyse Musicale, Paris, avril 1987, p. 16-21.

26. Guy Scarpetta, op. cit., p. 48. 
et des processus de lecture différents. En parcourant les différentes étapes du cheminement de Glenn Gould dans le texte des Variations Goldberg, nous avons décelé des caractéristiques qui permettraient de le définir en tant qu'“archilecteur 27 ". En effet, le processus au moyen duquel Gould produit du sens et organise la cohérence du texte comporte différents paliers de lecture de complexité croissante. Les étapes de ce processus sont les suivantes: lecture de la partition, construction de l'objet sonore fragment par fragment au moyen des techniques de l'enregistrement, production d'une notation de son interprétation et lecture des nouveaux signes avec renvoi à la partition du compositeur; lecture comparée avec les autres versions qu'il a déjà effectuées de la même œuvre. L'objectif de cette seconde partie consiste plus précisément à décrire et à identifier chacune de ces étapes.

Gould ne s'est pas contenté d'accumuler ou de collectionner ses différentes interprétations des Goldberg et il n'y a pas lieu en effet de le comparer au lecteur littéraire "ordinaire" qui, selon Gilles Thérien ${ }^{28}$, est "expert en extension". Ses deux enregistrements de 1955 et de 1981 conduisent plutôt à montrer que, dans son processus de lecture, il «reporte son acte de lecture à des catégories déjà retenues à partir d'autres actes de lecture mais organisés, structurés en systèmes ${ }^{29}$ ". En effet, chacune des

27. Ce concept a été appliqué à l'œuvre littéraire notamment par Michel Riffaterre, dans Essai de stylistique structurale, Paris, Flammarion, 1971, p. 46-48. «ll renvoie à un groupe d'informateurs utilisés pour chaque stimulus ou pour une séquence stylistique». Nous avons toutefois utilisé l'interprétation qu'en a donnée Gilles Thérien pouvant être adaptée à l'œuvre musicale.

28. Gilles Thérien, "Pour une sémiotique de la lecture", dans Protée, volume 18, numéro 2, printemps 1990, p. 67-80.

29. Ibid., p. 79. 
étapes de son processus s'effectue en fonction de présupposés théoriques au sujet de l'acte même de l'interprétation, de J. S. Bach, de l'œuvre elle-même, du principe de la variation chez Bach, Mozart, Beethoven, Brahms, et des autres interprétations des Goldberg. II importe d'identifier quelques pistes du savoir de Gould pour mieux comprendre ce à quoi renvoient ses différentes interventions en vue de la construction de sa propre représentation de l'œuvre.

Glenn Gould appartient à cette catégorie rare de musiciens-théoriciens qui, en tant qu'exécutants-interprètes, se soient intéressés à l'analyse et à la description du processus créateur en fonction d'une conception plus générale de l'art et de la musique. On sait qu'il se définissait interprète-compositeur, à la recherche de la signification intrinsèque de l'œuvre. Son idéal musical a toujours été fondé sur la raison et l'oreille, et il a su par conséquent se tourner vers des compositeurs qui ont accordé un rôle prépondérant au développement et à l'organisation des structures et des idées musicales. Parmi ces compositeurs figure J. S. Bach auquel il a réservé une place de choix dans son répertoire et dans ses écrits sur la musique :

[...] Bach constitue la raison première pour laquelle je suis devenu musicien ${ }^{30}[\ldots . .$.$] . C'est la structure en tant que telle qui$ signifie, indépendamment des sonorités que vous lui imposez $^{31}$.

II a su s'inspirer également du compositeur et théoricien Arnold Schoenberg. II lui a emprunté les principes de son esthétique formaliste de l'interprétation. S'il l'a autant admiré, c'est notamment pour avoir fait renaître la pensée contrapuntique issue de J. S. Bach. Gould est en effet attiré

30. Glenn Gould, Non, je ne suis pas du tout un excentrique, Paris, Fayard, 1986, p. 189.

31. Glenn Gould, Contrepoint à la ligne, op. cit., p. 34. 
par la musique polyphonique qui superpose plusieurs mélodies les unes aux autres, destinées à être entendues simultanément, sans qu'il y ait une priorité accordée à l'une ou à l'autre d'entre elles. Tout son répertoire repose sur des œuvres qui s'illustrent par l'explosion de leurs idées musicales simultanées. Mais, ce qui l'étonne encore chez «J. S. Bach, le non-conformiste ${ }^{32}$ », c'est la «sublime indifférence instrumentale" qui accompagne les préoccupations structurales de son œuvre. Gould utilise cette expression pour justifier son choix bien arrêté d'interpréter Bach au piano plutôt qu'au clavecin, afin de permettre un style d'articulation propre à exprimer avec plus de clarté la pensée contrapuntique du compositeur :

Je doute donc que, s'il avait connu le piano, il se soit beaucoup soucié de savoir si ce dernier dénaturait certaines intentions sonores spécifiques de sa musique ${ }^{33}$.

II refusera aussi de se réfugier derrière une tradition d'interprétation bachienne en ce qui concerne notamment le tempo, la dynamique de l'œuvre :

[...] Bach est structurellement si convaincant et ceci à tant de niveaux, on peut lui envisager tellement de situations selon des angles si différents $[\ldots]^{34}$.

Son intérêt pour une œuvre aussi complexe que les Goldberg sur le plan structural n'a donc rien d'étonnant. II est non seulement attaché au Bach architecte mais aussi au coloriste qui s'est appliqué dans cette œuvre à peindre différents tableaux aux caractères et aux textures diversifiés. II possède une connaissance du principe de la variation et une conception globale de l'œurre :

32. Titre d'un texte de Gould dans ibid., p. 12-20.

33. Glenn Gould, Contrepoint à la ligne, op. cit., p. 33.

34. Ibid., p. 26. 
Nous avons, également par l'analyse, observé qu'il n'est pas de séries de variations successives qui utilisent des principes de constructions similaires, tels que ceux qui donnent leur cohérence aux variations de Beethoven et de Brahms ${ }^{35}$.

Toutefois sa conception de l'œuvre dépasse les résultats qu'il a pu obtenir par une approche analytique :

[...] nous sentons bien, l'analyse n'étant ici d'aucun secours, qu'il existe là une intelligence coordinatrice fondamentale. [...]. Nous sommes dès lors contraint de réviser nos critères, lesquels sont simplement défectueux pour arbitrer cette union de la musique et de la métaphysique, qu'est le royaume de la transcendance technique ${ }^{38}$.

Il a également en mémoire différentes interprétations ${ }^{37}$ des Goldberg notamment celles de Rosalyn Tureck au clavecin; d'Antony Newman au clavecin également; de Peter Serkin au piano, et une interprétation pour musique de chambre par le Marlboro Festival du Vermont avec Rudolph Serkin à la direction de l'orchestre. II aurait été plus particulièrement influencé par l'interprétation de Rosalyn Tureck en ce qui concerne l'œuvre de Bach en général. Par ailleurs, il a toujours voulu aborder une partition sans idée préconçue quant à sa réalisation sonore. Néanmoins, la diversité des interprétations des Variations Goldberg ont dû contribuer à alimenter sa conviction à propos de «la sublime indifférence instrumentale» de Bach.

35. Ibid., p. 10.

36. Ibid.

37. Les enregistrements qui suivent apparaissent dans sa discographie personnelle : Rosalyn Tureck, Harpsichord, 1979, Columbia, M2-35900; Anthony Newman, Harpsichord, (no date), Columbia, M-3058; Peter Serkin, Piano, 1965, RCA Victor, LSC-2851; Marlboro Recording Society, (no number-no date), 14 Canons on the First Eight Bass Notes of the Aria Ground from the "Goldberg Variations", Rudolph Serkin, Piano with chamber groups. 
Tout en n'utilisant que le piano pour réaliser ses différentes versions des Variations, Gould démontre la possibilité d'établir, à chaque exécution, un nouveau rapport à l'œuvre et de l'aborder sous des angles différents. II a su en effet développer cette notion de différence propre à toute interprétation, à plusieurs niveaux, notamment, affirme Michel Schneider upar rapport aux autres pianistes, aux intentions des compositeurs, de la tradition, du bon sens, de soi-même antérieurement ${ }^{38}$ ". Or, ce travail de la différence qui découle des contextes de production, des conditions acoustiques, etc., suppose chez Gould, plusieurs exigences. Toute modification de l'œuvre, que ce soit aux niveaux des paramètres du rythme, du tempo, du phrasé, de l'ornementation, de l'accentuation, doit être fondée sur une conception préalable de la structure de l'œuvre et ne doit pas en altérer l'unité. Le studio de l'enregistrement plutôt que la salle de concert fournit les conditions essentielles à la poursuite de cette quête de l'interprétation idéale qui caractérise notamment les deux enregistrements de 1955 et de 1981. Le travail requis par cette quête suppose comme première étape la lecture et le décodage des signes de la partition.

Cette étape qui est celle de tout exécutant présente toutefois, dans le cas de Gould, un caractère particulier. En effet, Gould vise un idéal analytique de nature abstraite qui exclut, dans un premier temps, toute dimension tactile requise par la présence de l'instrument :

[...] ma technique est de passer le plus de temps possible loin de mon piano, ce qui pose certaines difficultés : on a souvent envie de savoir comment cela sonne. Mais un certain idéal analytique [...], un certain achèvement analytique quoi qu'il en soit est théoriquement possible tant que vous n'êtes pas au

38. Michel Schneider, Glenn Gould, Piano solo, Paris, Gallimard, 1988, p. 96-97. 
piano. Lorsque vous allez au piano, les compromissions tactiles que vous êtes amenés à faire limitent cette perfection ${ }^{30}$.

Gould «joue» avec les éléments qui composent la structure de chacune des variations, les organise, les transforme, les déplace, les regroupe, les compare. Il se penche par exemple sur les préoccupations contrapuntiques des variations canoniques et sur les "cellules thématiques" des variations de caractère indépendant comme l'Ouverture, l'Alla Breve, le Quodlibet. La $25^{\circ}$ variation en sol mineur "cette indolente et mélancolique cantilène 40 " le conduit à interroger la position stratégique qu'elle occupe par rapport à l'ensemble des variations. Enfin, l'analyse lui démontre que :

[...] la mélodie de l'Aria [...], évite tout commerce avec le reste de l'œuvre, chaque variation consomme avec voracité le potentiel du germe mélodique qui lui est particulier $[. . .]^{41}$.

Cette première approche théorique le conduit par la suite à la construction de l'objet sonore, par le biais des techniques d'enregistrement. En d'autres termes, il soumet cette fois son approche théorique à l'épreuve de la dimension tactile. C'est alors la reprise, variation par variation, fragment par fragment, jusqu'à satisfaction du sens obtenu pour tel phrasé, tel ornement, tel accent. Le document sonore ${ }^{42}$ des prises ou "out takes" ayant servi à la version de 1981 démontre que cette recherche est sans limite. Trente prises pour deux mesures de la variation 9; quinze

39. Glenn Gould, Entretiens avec Jonathan Cott, traduit et présenté par Jacques Drillon, Paris, J.C. Lattès, 1983, p. 59.

40. Glenn Gould, Contrepoint à la ligne, op. cit., p. 10.

41. Ibid., p. 8.

42. II s'agit d'un document inédit disponible au Fonds Glenn Gould, Bibliothèque nationale du Canada, Division de la musique, Ottawa. 
prises pour l'ornement du début de l'Aria. Autant de prises, autant de représentations possibles de la variation ou du fragment qui, mis bout à bout, moyennant les techniques de découpage et de collage, formeront un tout cohérent, comme il en est du montage d'un film.

Il est possible aussi de constater dans ce même matériel une autre dimension plus émotive et plus affective qui vient compléter l'approche intellectuelle de la partition. Gould, en effet, se montre parfois bavard, expliquant pourquoi il aime ou pas tel passage, tel fragment qu'il vient d'exécuter. II lui arrive d'exprimer des cris de joie, d'enthousiasme mais de colère aussi. Et, à des moments plus rares, lorsque l'expression se fait débordante, c'est le chant qui remplace les notes, afin d'exprimer cet excès de musique qu'il écoute en lui-même et pour lui-même. L'extase se fait toutefois plus discrète et fait place à la seule musique !

Cette même interprétation de 1981 a donné lieu, comme l'illustre l'exemple à la page 98, à un autre document qui permet de constater l'extrême contrôle que Gould a toujours voulu exercer sur son interprétation. II s'agit cette fois de la production d'une nouvelle notation ou de nouveaux signes qui renvoient à la lecture de son interprétation et concernent plus spécifiquement les nuances, le timbre, le phrasé de chaque variation. 
$\operatorname{Van}$ II

Man dynarie cunves needaristimo (I) $t$ on Bon 16 Danteid s frocth

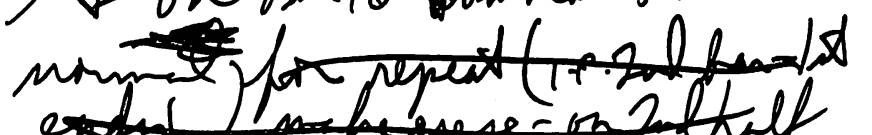

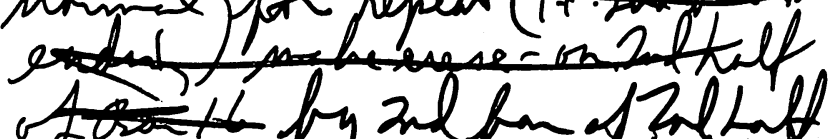

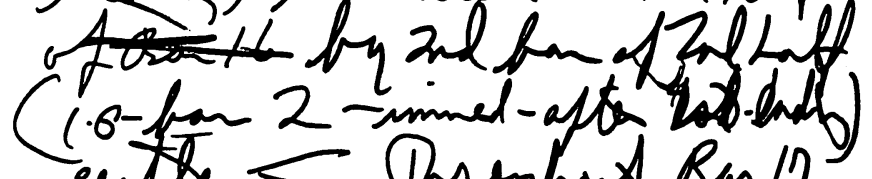

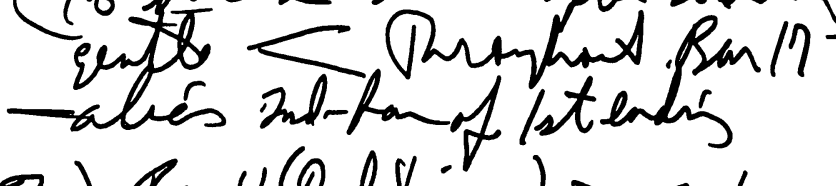

(2) Sen $4(21$ ling areon-elt hise - The (E) is

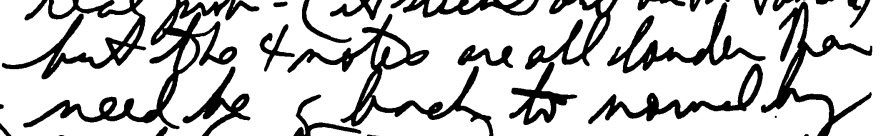

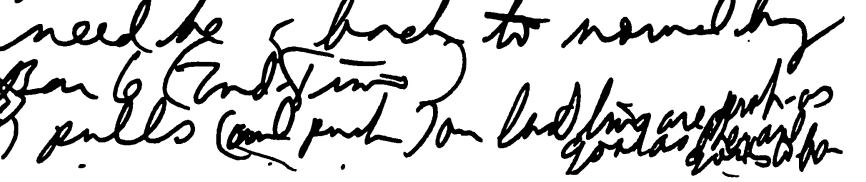
- (4) ald slifts ton 32023

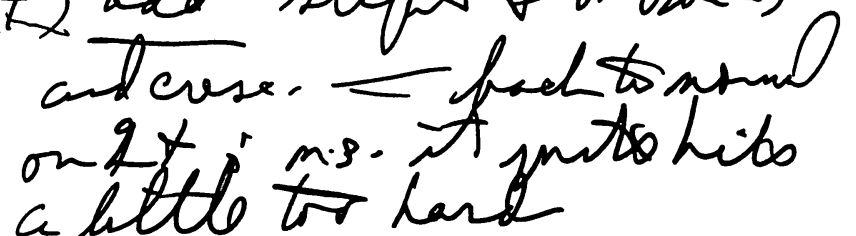
c little to hard

98 


\section{Var. VI}

Many dynamic curves need assistance.

1) $\downarrow$ on Bar 16. Downbeat and back to normal, for repeat (i.e. 2nd bar-1st ending) make cresc (endo) on 2 nd half of Bar 16 by 2 nd bar of 2 nd half (i.e. bar 2-immed (iately) - after 1st ending) gentle < throughout. Bar 17 - alies (sic) 2nd bar of 1st ending

2) Bar 4 (2nd time) $>$ to accom (pany) alto line; the $(\xi)$ is the real probl (lem) - (it sticks out on the $\longrightarrow$ but the 4 notes are all louder than need be; back to normal by Bar 6 (2nd time)

3) pulls (and push) on last line are prob (ably) as good as they are going to be.

4) add slight _ on Bar 23 and cresc (endo) < back to normal on 2 n.s. it quits hits a little too hard ${ }^{43}$.

Tout porte à croire, si l'on se réfère à la réflexion qui suit, que la lecture de cette nouvelle partition a été accompagnée d'une autre lecture en référence cette fois à la partition du compositeur :

II s'agit là d'une pratique très vulnérable, surtout appliquée à la musique de Bach, qui ne concède guère d'indications de tempo ou de dynamique, et je dois me mettre moi-même en garde contre le danger qu'il y a à se laisser aller à confondre l'enthousiasme d'une conviction interprétative et l'absolu inaltérable de la volonté d'un compositeur ${ }^{44}$.

43. Ce document, extrait du journal de Gould, est inédit. II est disponible au Fonds Glenn Gould, Bibliothèque nationale du Canada, Division de la musique, Ottawa. Nous remercions la succession de Glenn Gould pour son aimable collaboration, et notre assistante de recherche Valerie Verity, pour avoir tenté de déchiffrer l'écriture de Gould.

44. Glenn Gould, Contrepoint à la ligne, op. cit., p. 6. 
Une dernière étape vient compléter cette lecture. Gould comparera sa nouvelle lecture de l'œuvre avec celles qu'il avait actualisées dans le passé. II y puisera des matériaux qu'il conservera, modifiera, ajoutera à sa nouvelle lecture. Le Gould théoricien, écrivain, a fait connaître les résultats de sa démarche comparative, notamment à propos de ses versions de 1955 et de 1981. II affirme ne pas reconnaître la personne spirituelle qui a fait l'enregistrement de 1955. S'il n'est pas sans apprécier l'humour de l'interprétation de 55, il demeure néanmoins étranger à la manière dont il concevait l'enregistrement à cette époque :

[...] ]'étais avant tout un artiste de concert, et il s'ensuivait que je m'efforçals de reproduire en studio ce que je pouvais faire de mieux en concert [...] j'envisage maintenant l'acte d'enregistrement comme quelque chose de tout à fait spécial qui n'a plus rien à voir avec la reproduction d'aucun autre acte ${ }^{45}$.

Gould ira encore plus loin dans sa comparaison, en prenant comme exemple l'interprétation de

la quinzième variation, très longue et très lente, qui est un canon inversé à la quinte. Il y a vingt-six ans, je lui avais donné l'allure d'un Nocturne de Chopin [...]. Aujourd'hui, cette pièce est possédée, pour moi, d'une intensité dépourvue de tout effet extérieur ${ }^{48}$.

En 1981, il optera pour une plus grande distanciation vis-à-vis de son instrument (un piano Yamaha). II cherchera à obtenir une plus grande continuité entre les variations, visant ainsi l'aspect monolithique de l'œuvre. II accentuera les contrastes en utilisant une technique différente dans l'interprétation des reprises de la première partie des canons. En 1955, sur un piano Steinway, il fera, de chacune

45. Glenn Gould, Non, je ne suis du tout un excentrique, Paris, Fayard, 1986, p. 172.

46. Ibid. 
des variations, de véritables miniatures, sans enchaînement, sans reprise, sans le souci d'unification présent de 1981. Cependant mieux vaut encore laisser la parole à Gould qui précise une toute dernière fois la comparaison entre ses deux versions :

I also recog(nize) that many people will prefer this early version, that many people will find the new one rather stark and spare emotionally. But this represents everything that I personally object to in the early version - it wears its heart on its sleeve; it says please note, this is not of tragedy, you know, it doesn't have the dignity to bear its suffering with a hint of quiet resignation ${ }^{47}$.

Comment ne pas vouloir conclure sur l'écoute de l'Aria de la dernière version. Une écoute qui saura nous faire oublier que le thème à la basse, si anodin, si ingénu, constitue dans cet enregistrement la prise 21. La technologie est ici au service du sens de l'œuvre. II revient à la sémiologie de le décoder, de l'interpréter. Une approche tout à fait conforme à la nature même de la musique qui, en tant qu'art de l'interprétation, présente un terrain propice pour comprendre les exigences d'un tel processus créateur.

Le Glenn Gould, “archilecteur» des Goldberg, a démontré les possibilités d'application de ce concept à un autre domaine que celui de la littérature. Cette approche a permis d'identifier différents niveaux de complexité dans la lecture d'un "texte" musical par un interprète. Encore faudrait-il, suite à la problématique que nous avons à peine esquissée, identifier les résultats de chacune des étapes de la lecture de Gould, voir de plus près les données de sa représentation de l'œuvre, définir le système de signes auquel elle renvoie. L'ensemble de cette démarche em-

47. Glenn Gould, extrait du manuscrit de l'entrevue avec Tim Page, édité chez CBS Records Masterw, CDN-70, IM 37779, août 1982. 
pruntée à l'analyse sémiologique demeure également ouverte à l'analyse d'un autre corpus d'œuvres et d'interprètes. Elle conduirait ainsi à définir d'autres types et processus de lecture et, partant, contribuerait à une meilleure compréhension de la nature de l'interprétation musicale.

Ghyslaine Guertin Collège Édouard-Montpetit 